\title{
Use of Early Goal-Directed Therapy in the Emergency Department before and after the Sepsis Trilogy
}

\author{
Loren K. Reed, Benton R. Hunter, Tyler M. Stepsis \\ Department of Emergency Medicine, Indiana University School of Medicine, Indiana University, Indianapolis, IN, \\ USA \\ Email: loreed@iu.edu
}

Received 24 March 2016; accepted 10 June 2016; published 13 June 2016

Copyright (C) 2016 by authors and Scientific Research Publishing Inc.

This work is licensed under the Creative Commons Attribution International License (CC BY). http://creativecommons.org/licenses/by/4.0/

(c) (i) Open Access

\section{Abstract}

The management of sepsis evolved recently with the publication of three large trials (referred to as the sepsis trilogy) investigating the efficacy of early goal-directed therapy (EGDT). Our goal was to determine if the publication of these trials has influenced the use of EGDT when caring for patients with severe sepsis and septic shock in the emergency department (ED). In February 2014, we surveyed a sample of board-certified emergency medicine physicians regarding their use of EGDT in the ED. A follow-up survey was sent after the publication of the sepsis trilogy. Data was analyzed using $95 \%$ confidence intervals to determine if there was a change in the use of EGDT following the publication of the above trials. Subgroup analyses were also performed with regard to academic affiliation and emergency department volume. Surveys were sent to 308 and 350 physicians in the pre-and post-publication periods, respectively. Overall, ED use of EGDT did not change with publication of the sepsis trilogy, $48.7 \%$ (CI $39.3 \%-58.2 \%$ ) before and $50.5 \%$ (CI $40.6 \%-60.3 \%$ ) after. Subgroup analysis revealed that academic-affiliated EDs significantly decreased EGDT use following the sepsis trilogy while nonacademic departments significantly increased EGDT use. Use of EGDT was significantly greater in community departments versus academic departments following the publication of the sepsis trilogy. There was no change overall in the use of EGDT protocols when caring for patients with severe sepsis and septic shock, but subgroup analyses revealed that academic departments decreased their use of EGDT while community departments increased use of EGDT. This may be due to varying rates of uptake of the medical literature between academic and community healthcare systems.

\section{Keywords}

Sepsis, Early Goal-Directed Therapy, Septic Shock, EGDT 


\section{Introduction}

Severe sepsis is commonly encountered in the emergency department (ED). Over 500,000 patients with severe sepsis or septic shock are cared for in the ED every year, spending an average of 5 hours there prior to admission [1]. In 2001, a protocol, "Early Goal-Directed Therapy" (EGDT) was found, in a single randomized trial by Rivers et al., to result in a substantial decrease in mortality among patients with severe sepsis (absolute risk reduction 16\%) [2]. Several subsequent observational studies corroborated these findings [3] [4].

EGDT, which is based on using objective endpoints to maximize tissue oxygenation, is both a time and resource intensive process that requires close monitoring of central venous pressure (CVP), central venous oxygen saturation (ScVO2), and mean arterial pressure (MAP), thus necessitating invasive procedures for hemodynamic monitoring. While guidelines have encouraged the use of EGDT, and many EDs invested in the equipment and resources required to implement it, some EDs were unwilling or unable to enact EGDT according to the strict parameters outlined in Rivers' study. Many have argued that the benefits of EGDT are not due to achieving these strict hemodynamic endpoints, but simply early recognition and aggressive resuscitation of these very sick patients.

Recently, this question has been specifically addressed by three large randomized multi-center trials, referred to as the "sepsis trilogy", which included the ProCESS trial published in May 2014, the ARISE trial published in October 2014, and the ProMISe trial published in April 2015 [5]-[7]. Individually and cumulatively these studies demonstrated that a strict protocolized approach to the care of septic patients was not superior to "standard care" with aggressive early resuscitation.

The purpose of our study was to determine if the publication of the recent sepsis trilogy has influenced the use of protocol-based EGDT in the ED. We surveyed a sample of board-certified emergency medicine physicians regarding the use of protocol-based EGDT before and after the publication of the sepsis trilogy. Given the findings of the trials, we hypothesized that the use of protocol-based EGDT would decrease after their publication.

\section{Methods}

The initial survey regarding the use of EGDT in the ED was sent in February of 2014 to our departmental and residency alumni listserv, which, at the time, consisted of 308 board-certified emergency physicians practicing in over 30 states. Responses were collected through the end of March 2014. A follow-up survey was then conducted during August and September of 2015 following the publication of the sepsis trilogy. At this time, the listserv consisted of 350 physicians. The surveys were constructed using an online survey tool and disseminated by electronic mail to the participants. Demographic data was collected and subgroup analyses were performed. Chi-square tests of independence were used to ensure no difference in demographics was present between initial and follow-up respondents. We specifically analyzed academic and community EDs separately as well as large EDs (defined as patient volume $>50,000$ visits per year) and small EDs (defined as patient volume $\leq 50,000$ visits per year). Point estimates of population proportions were compared with exact binomial 95\% confidence intervals. Significant difference was assumed when the compared proportion was not contained in the $95 \%$ confidence interval.

\section{Results}

We received 115 responses (37\%) to the initial survey and 107 responses (31\%) to the follow-up survey. As detailed in Table 1, the respondent group demographics were similar with respect to ED academic affiliation $(\mathrm{p}=$ $0.922)$, location ( $p=0.624)$, and volume $(p=0.193)$. The most common barriers to the use of EGDT included the need for specialty equipment such as continuous ScVO2 monitoring (36.5\%) and central venous cannulation (25.5\%).

As seen in Figure 1, overall there was no significant change in the use of EGDT protocols with 48.7\% (CI $39.3 \%$ - 58.2\%) use before and 50.5\% (CI 40.6\% - 60.3\%) following publication of the sepsis trilogy. Subgroup analyses revealed a significant decrease in use of EGDT in academic EDs with 56.3\% (CI 41.2\% - 70.5\%) use initially and 39.3\% (CI 25.0\% - 55.6\%) on follow-up. In nonacademic departments, EGDT use actually increased significantly from $43.3 \%$ (CI 31.2\% - 56.0\%) to 57.8\% (CI 44.8\% - 70.1\%). Analysis of EDs with respect to patient volume demonstrated no significant change in EGDT usage. Large department EGDT usage was $52.2 \%$ (CI 39.7\% - 64.6\%) before and 49.2\% (CI 36.1\% - 62.3\%) after publication of the sepsis trilogy, while 


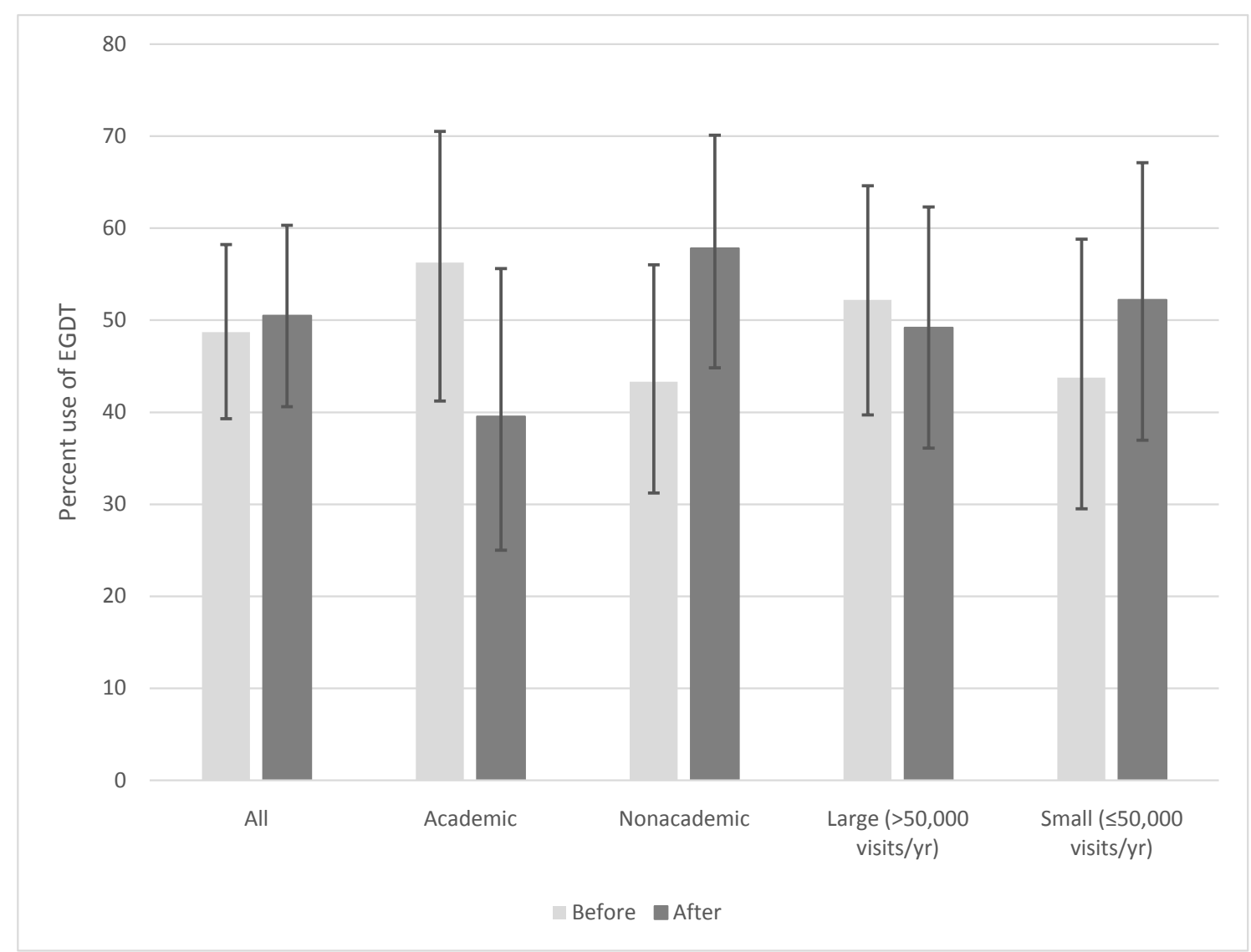

Figure 1. Use of Early Goal Directed Therapy (EGDT) before and after the publication of the sepsis trilogy. The percent of respondents reporting use of EGDT in their emergency departments before and after publication of the ProCESS, ProMISe, and ARISE trials is shown with accompanying 95\% confidence intervals. All respondents ( $\mathrm{n}=$ 115 before and $n=107$ after) as well as academic ( $n=48$ before and $n=43$ after), nonacademic $(n=67$ before and $n$ $=64$ after), large ED ( $n=67$ before and $n=61$ after), and small ED ( $n=48$ before and $n=46$ after) subgroups are included.

Table 1. Survey respondent demographics. Demographic data was collected from respondents to the initial and follow-up surveys. There was no difference between groups of respondents (emergency department type $p=0.922$, emergency department location $\mathrm{p}=0.624$, emergency department volume $\mathrm{p}=0.193)$.

\begin{tabular}{|c|c|c|c|}
\hline & & Initial & Follow up \\
\hline \multirow[t]{2}{*}{ Emergency Department Type } & Academic & 48 & 43 \\
\hline & Community & 67 & 64 \\
\hline \multirow[t]{3}{*}{ Emergency Department Location } & Urban & 46 & 50 \\
\hline & Suburban & 58 & 46 \\
\hline & Rural & 11 & 11 \\
\hline \multirow[t]{11}{*}{ Emergency Department Volume } & $<10,000$ & 3 & 0 \\
\hline & $10,001-20,000$ & 1 & 3 \\
\hline & $20,001-30,000$ & 19 & 12 \\
\hline & $30,001-40,000$ & 15 & 15 \\
\hline & $40,001-50,000$ & 10 & 16 \\
\hline & $50,001-60,000$ & 16 & 8 \\
\hline & $60,001-70,000$ & 18 & 11 \\
\hline & $70,001-80,000$ & 11 & 16 \\
\hline & $80,001-90,000$ & 4 & 4 \\
\hline & $90,001-100,000$ & 6 & 4 \\
\hline & $>100,000$ & 12 & 18 \\
\hline
\end{tabular}


small department usage was 43.8\% (CI 29.5\% - 58.8\%) before and 52.2\% (CI 36.7\% - 67.1\%) after. Prior to publication of the sepsis trilogy, there was no significant difference in use of EGDT between academic and nonacademic EDs. Following the sepsis trilogy, EGDT use in nonacademic EDs was significantly greater than in academic EDs, 57.8\% (CI 44.8\% - 70.1\%) versus 39.5\% (CI 25.0\% - 55.6\%).

\section{Discussion}

Since the original EGDT publication by Rivers, et al. in 2001, [2] there has been substantial debate about whether the observed benefit was due to strict adherence to the EGDT protocol or was simply the result of the early and aggressive resuscitation. Since that time, "standard care" has evolved to reflect an understanding that patients with severe sepsis and septic shock need early aggressive resuscitation and antibiotics. The results from the ProCESS, ProMISe, and ARISE trials suggest that the rigid measures of EGDT are not superior to usual care with aggressive fluid resuscitation seen in today's EDs [5]-[7].

As revealed in our results and elsewhere, the most significant barriers to the use of EGDT remain the need for specialty equipment and utilization of too many ED resources for implementation and maintenance [8]-[10]. The results of the sepsis trilogy obviate many of the above barriers.

Despite two years having passed since the publication of the ProCESS trial, overall the use of strict EGDT has not significantly changed among our survey respondents. However, our subgroup analysis revealed that following the sepsis trilogy there was a significant decrease in the use of EGDT at academic centers and a significant increase at community centers. Previous studies have estimated that the translation of original research into standard clinical practice takes approximately 17 years [11]. Our data suggests that, particularly in non-academic EDs, the use of EGDT may still be increasing, now 14 years after Rivers’ original trial.

After the publication of these trials, academic centers appear to have changed their practice in a manner consistent with the findings of the sepsis trilogy, while community departments have done the opposite. To our knowledge, there is no literature comparing the speed with which academic and community hospitals adopt new research into practice. Further research in this area is warranted.

\section{Limitations}

There are several limitations to our study. Only 31\% and 37\% of those surveyed responded, though this is consistent with the expected response rate for similar surveys. The use of EGDT was self-reported and not objectively measured. Additionally, the listserv that our survey was disseminated to consists mostly of emergency physicians who graduated from the same emergency medicine residency and may thus tend toward similar practice patterns. Our survey asked about departmental use of EGDT, as opposed to individual practice, which may minimize this limitation. Lastly, respondents to the second survey we sent were not necessarily the same as those who responded to the initial survey, so changes in reported use of EGDT do not necessarily reflect changes in any individual physician or department practice.

\section{Conclusion}

Respondents to our survey overall have not decreased their use of EGDT for severe sepsis following the publication of the ProCESS, ProMISe, and ARISE trials [5]-[7]. Those working in academic EDs decreased the use of EGDT following the sepsis trilogy while those working in non-academic EDs increased the use of EGDT.

\section{References}

[1] Wang, H.E., Shapiro, N.I., Angus, D.C. and Yealy, D.M. (2007) National Estimates of Severe Sepsis in United States Emergency Departments. Critical Care Medicine, 35, 1928-1936.

http://dx.doi.org/10.1097/01.CCM.0000277043.85378.C1

[2] Rivers, E., Nguyen, B., Havstad, S., Ressler, J., Muzzin, A., Knolich, B., et al. (2001) Early Goal-Directed Therapy in the Treatment of Severe Sepsis and Septic Shock. The New England Journal of Medicine, 345, 1368-1377. http://dx.doi.org/10.1056/NEJMoa010307

[3] Jones, A.E., Focht, A., Horton, J.M. and Kline, J.A. (2007) Prospective External Validation of the Clinical Effectiveness of an Emergency Department-Based Early Goal-Directed Therapy Protocol for Severe Sepsis and Septic Shock. Chest, 132, 425-432. http://dx.doi.org/10.1378/chest.07-0234 
[4] Jones, A.E., Brown, M.D., Trzeciak, S., Shapiro, N.I., Garrett, J.S., Heffner, A.C., et al. (2008) The Effect of a Quantitative Resuscitation Strategy on Mortality in Patients with Sepsis: A Meta-Analysis. Critical Care Medicine, 36, 2734-2739. http://dx.doi.org/10.1097/CCM.0b013e318186f839

[5] Yealy, D.M., Kellum, J.A., Huang, D.T., Barnato, A.E., Weissfeld, L.A., Pike, F., et al. (2014) A Randomized Trial of Protocol-Based Care for Early Septic Shock. The New England Journal of Medicine, 370, 1683-1693. http://dx.doi.org/10.1056/NEJMoa1401602

[6] Peake, S.L., Delaney, A., Bailey, M., Bellomo, R., Cameron, P.A., Cooper, D.J., et al. (2014) Goal-Directed Resuscitation for Patients with Early Septic Shock. The New England Journal of Medicine, 371, 1496-1506. http://dx.doi.org/10.1056/NEJMoa1404380

[7] Mouncey, P.R., Osborn, T.M., Power, S., Harrison, D.A., Sadique, Z., Grieve, R.D., et al. (2015) Trial of Early, Goal-Directed Resuscitation for Septic Shock. The New England Journal of Medicine, 374, 1301-1311. http://dx.doi.org/10.1056/NEJMoa1500896

[8] Jones, A.E. and Kline, J.A. (2005) Use of Goal-Directed Therapy for Severe Sepsis and Septic Shock in Academic Emergency Departments. Critical Care Medicine, 33, 1888-1890. http://dx.doi.org/10.1097/01.CCM.0000166872.78449.B1

[9] Carlbom, D.J. and Rubenfeld, G.D. (2007) Barriers to Implementing Protocol-Based Sepsis Resuscitation in the Emergency Department-Results of a National Survey. Critical Care Medicine, 35, 2525-2532. http://dx.doi.org/10.1097/01.ccm.0000298122.49245.d7

[10] Turi, S.K. and Von Ah, D. (2013) Implementation of Early Goal-Directed Therapy for Septic Patients in the Emergency Department: A Review of the Literature. Journal of Emergency Nursing, 39, 13-19. http://dx.doi.org/10.1016/j.jen.2011.06.006

[11] Balas, E.A. (1998) From Appropriate Care to Evidence-Based Medicine. Pediatric Annals, 27, 581-584. http://dx.doi.org/10.3928/0090-4481-19980901-11 